\title{
Discrimination of Tumorous Intracerebral Hemorrhage from Benign Causes Using CT Densitometry
}

\author{
(D)Y.S. Choi, T.H. Rim, S.S. Ahn, and S.-K. Lee
}

\begin{abstract}
BACKGROUND AND PURPOSE: Differentiation of tumorous intracerebral hemorrhage from benign etiology is critical in initial treatment plan and prognosis. Our aim was to investigate the diagnostic value of CT densitometry to discriminate tumorous and nontumorous causes of acute intracerebral hemorrhage.
\end{abstract}

MATERIALS AND METHODS: This retrospective study included 110 patients with acute intracerebral hemorrhage classified into 5 groups: primary intracerebral hemorrhage without (group 1) or with antithrombotics (group 2) and secondary intracerebral hemorrhage with vascular malformation (group 3), brain metastases (group 4), or primary brain tumors (group 5). The 5 groups were dichotomized into tumorous (groups 4 and 5) and nontumorous intracerebral hemorrhage (groups 1-3). Histogram parameters of hematoma attenuation on nonenhanced CT were compared among the groups and between tumorous and nontumorous intracerebral hemorrhages. With receiver operating characteristic analysis, optimal cutoffs and area under the curve were calculated for discriminating tumorous and nontumorous intracerebral hemorrhages.

RESULTS: Histogram analysis of acute intracerebral hemorrhage attenuation showed that group 1 had higher mean, 5th, 25th, 50th, and 75th percentile values than groups 4 and 5 and higher minimum and 5th percentile values than group 2 . Group 3 had higher 5 th percentile values than groups 4 and 5 . After dichotomization, all histogram parameters except maximum and kurtosis were different between tumorous and nontumorous intracerebral hemorrhages, with tumors having lower cumulative histogram parameters and positive skewness. In receiver operating characteristic analysis, 5th and 25th percentile values showed the highest diagnostic performance for discriminating tumorous and nontumorous intracerebral hemorrhages, with 0.81 area under the curve, cutoffs of $34 \mathrm{HU}$ and $44 \mathrm{HU}$, sensitivities of $65.6 \%$ and $70.0 \%$, and specificities of $85.0 \%$ and $80.0 \%$, respectively.

CONCLUSIONS: CT densitometry of intracerebral hemorrhage on nonenhanced CT might be useful for discriminating tumorous and nontumorous causes of acute intracerebral hemorrhage.

ABBREVIATIONS: $I C H=$ intracerebral hemorrhage; $\mathrm{NECT}=$ nonenhanced $\mathrm{CT}$

$\mathrm{T}$ he incidence of intracerebral hemorrhage (ICH) is $10-20$ cases per 100,000 worldwide. ${ }^{1-3} \mathrm{ICH}$ has a high mortality rate with case-fatality rates of $34.6 \%$ at 7 days and $59.0 \%$ at 1 year. ${ }^{4}$ Intracerebral hemorrhage is classified as either primary or secondary on the basis of the underlying cause of bleeding. Primary $\mathrm{ICH}$ accounts for $78 \%-88 \%$ of ICH cases and is caused by spontaneous rupture of small vessels associated with chronic hyperten-

Received September 5, 2014; accepted after revision November 9.

From the Department of Radiology and Research Institute of Radiological Science (Y.S.C., S.S.A., S.-K.L.) and Department of Ophthalmology, Institute of Vision Research (T.H.R.), College of Medicine, Yonsei University, Seoul, Korea.

Please address correspondence to Seung-Koo Lee, MD, PhD, Department of Radiology, College of Medicine, Yonsei University, 50 Yonsei-ro, Seodaemun-gu, Seoul 120-752, Korea; e-mail: SLEE@yuhs.ac

- Indicates open access to non-subscribers at www.ajnr.org

http://dx.doi.org/10.3174/ajnr.A4233 sion or amyloid angiopathy. ${ }^{5}$ Secondary ICH can occur in association with vascular malformations or tumors. ${ }^{1}$ Tumors account for $10 \%$ of all spontaneous ICH cases. ${ }^{6,7}$ Earlier discrimination of tumorous etiology from nontumorous causes of acute ICH might be helpful to determine appropriate imaging follow-up and avoid delays in long-term management of tumorous $\mathrm{ICH}$, though acute management would not differ.

Nonenhanced CT (NECT) is the standard investigation tool for acute ICH. Because most patients with $\mathrm{ICH}$ with tumors have a solid enhancing portion, contrast-enhanced CT or contrast-enhanced MR imaging are indicated when secondary ICH with tumor is suspected. ${ }^{8-10}$ However, enhancement of secondary ICH can be obscured by surrounding high attenuation or various T1 signal intensities from hematoma; these make diagnosis of secondary ICH difficult. ${ }^{10-12}$ Dual-energy CT offers better differentiation between ICH of tumor and nontumor etiology because of better visualization 


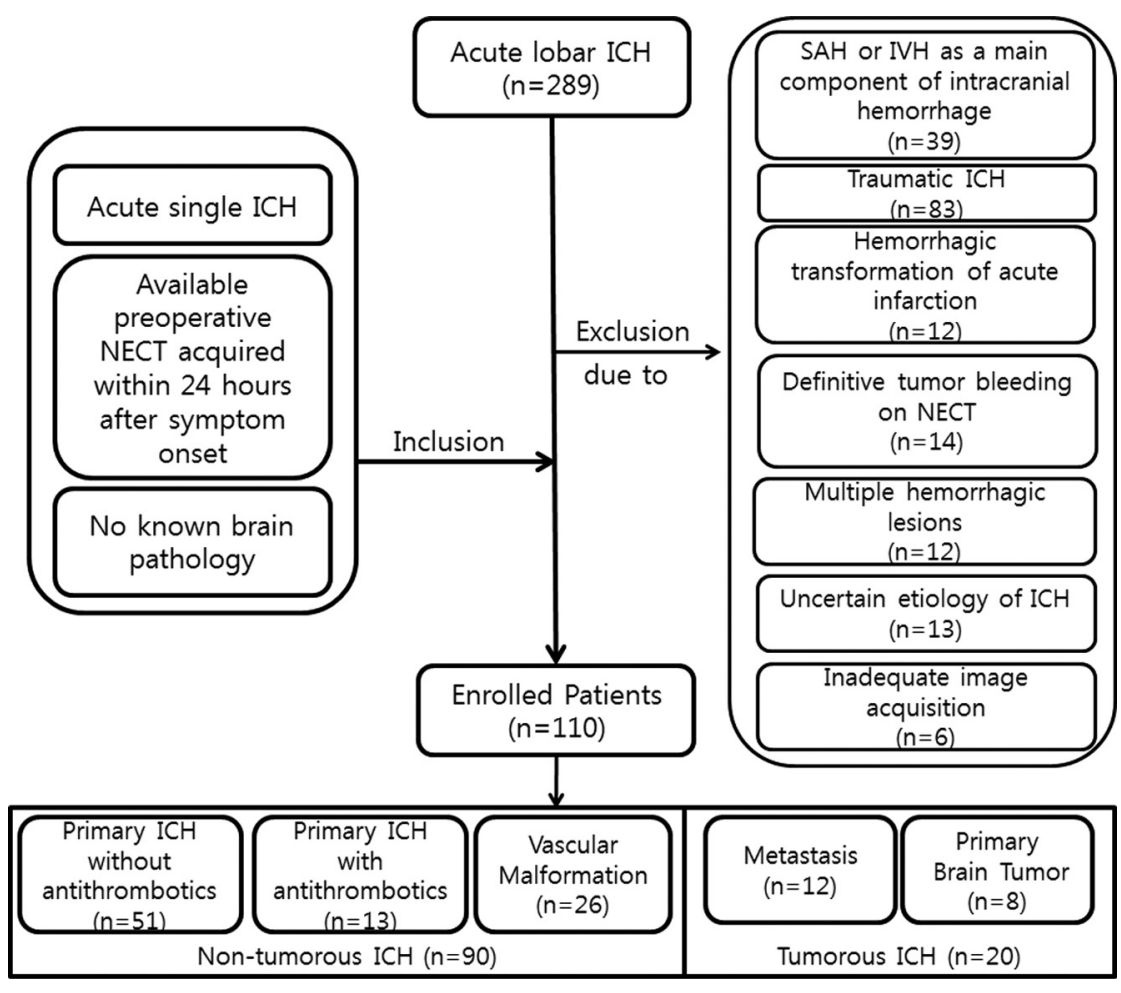

FIG 1. Flowchart of study population enrollment. IVH indicates intraventricular hemorrhage. with initial outpatient NECT images were included if they met the inclusion criteria and had images of adequate quality. To include clinically confusing patients only, we excluded patients with clinical or NECT imaging findings implicating certain underlying causes of $\mathrm{ICH}$, either primary or secondary. Exclusion criteria consisted of intraventricular hemorrhage or $\mathrm{SAH}$ as a main component of intracranial hemorrhage $(n=39)$, traumatic ICH $(n=83)$, or hemorrhagic transformation of acute infarction $(n=12)$, and patients with definitive tumorous ICH with gross cystic or solid portions $(n=14)$ or multiple hemorrhagic lesions $(n=12){ }^{9,15}$ Additionally, we also excluded cases of uncertain-cause ICH in patients who were either not sufficiently followed up with imaging or whose condition was not pathologically proved and could not be retrospectively classified as tumorous or nontumorous ICH $(n=13)$. Patients with initial outpatient NECT images of inadequate image quality were also excluded $(n=6)$.

The study cohort of 110 patients was classified into 5 groups according to underlying causes and antithrombotic medication status: 1) primary ICH and not taking antithrombotics; 2) primary ICH with antithrombotic use within 3 months before the CT scan; 3) secondary ICH due to vascular malformation such as AVM, cavernous angioma, or dural arteriovenous fistula; 4) secondary ICH due to brain metastases; and 5) secondary ICH due to primary brain tumors.

These 5 groups were later combined and dichotomized into patients with nontumorous ICH (groups 1, 2, and 3) and patients with tumorous ICH (groups 4 and 5).

\section{Image Acquisition and Analysis}

Among the 110 patients, the CT scans of 67 were obtained in our hospital. CT examinations were performed with voltage from 100 to $120 \mathrm{kV}$ and current from 130 to $250 \mathrm{~mA}$. CT scans of the other 43 patients were performed at other hospitals, with voltage from 100 to $120 \mathrm{kV}$ and current from 100 to $300 \mathrm{~mA}$.

Image analysis was by consensus by 2 neuroradiologists (Y.S.C., with 6 years of experience in brain CT reading; and S.-K.L., with 24 years of experience in brain CT reading) who were blinded to the clinical information. An ROI was drawn on all NECT axial images containing the entire ICH volume by using a semiautomatic method, with thresholding of Hounsfield unit values as described elsewhere ${ }^{16}$ and depicted in Fig 2. For drawing ROIs, a lower limit of 25-40 HU and an upper limit of $130 \mathrm{HU}$ were used as thresholds. For ICH adjacent to the skull, skull stripping was performed before drawing the ROI by masking pixels higher than $130 \mathrm{HU}$. Image analysis used the Medical Image Processing, Analysis, and Visualization software package (Version 7.0; National Institutes of Health, Bethesda, Maryland). 


\section{Statistical Analysis}

Demographic data were compared among the 5 groups by using 1 -way analysis of variance for continuous data and a $\chi^{2}$ test for categoric data. Histogram parameters were extracted from Hounsfield unit values of ROIs, which were minimum, 5th, 25th, 50th, 75th, 95th percentiles, maximum values, skewness, and kurtosis. Histogram parameters were compared among the 5 groups by using 1-way analysis of variance with post hoc pair-wise comparison with Bonferroni correction of $P$ values. To confirm the association between hematoma attenuation and time interval from
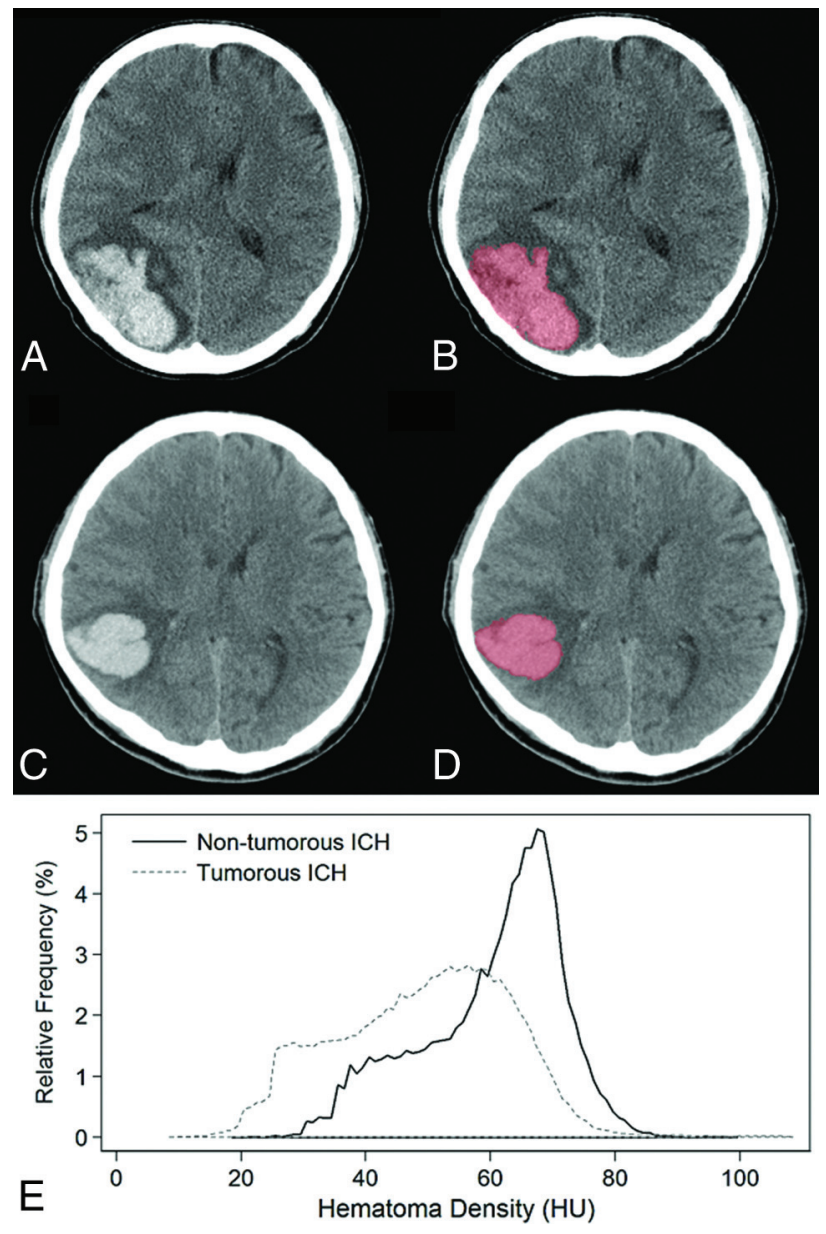

FIG 2. Representative cases of semiautomatic segmentation of tumorous $(A$ and $B$ ) and nontumorous $(C$ and $D) \mathrm{ICH}$ s and their relative frequency histogram of hematoma attenuation $(E)$. ICH was segmented with a semiautomatic method based on a voxel-intensity threshold of 40-130 HU. On histogram analysis, the 5th and 25th percentile values were $26 \mathrm{HU}$ and $38 \mathrm{HU}$ in tumorous ICHs and $40 \mathrm{HU}$ and $65 \mathrm{HU}$ in nontumorous $\mathrm{ICHs}$, respectively, and were discriminated correctly by using our histogram analysis. symptom onset to CT scan, we used multivariate linear regression after adjusting for possible confounders, including age and sex.

Histogram parameters were compared by $t$ test or nonparametric Wilcoxon rank sum test between tumorous and nontumorous ICHs after dichotomization of the study population according to the results of the Shapiro-Wilk test for normalized distribution.

Receiver operating characteristic analysis was performed to determine the optimal cutoff values for histogram parameters to discriminate tumorous and nontumorous ICHs. The optimal cutoff value was determined by maximizing the Youden index and calculating the area under the curve. All calculated $P$ values were 2 -sided, and results were considered significant if the $P$ value was $<.05$. We performed statistical analysis with STATA 12.1 (StataCorp, College Station, Texas).

\section{RESULTS}

\section{Study Population}

Demographic data of the study population are in Table 1. Among the 110 study patients (mean age, 53.4 years; range, 2.7-88.1 years), 64 had primary ICH and were classified in groups 1 or 2 . Thirteen of 64 were classified as group 2, consisting of 6 patients taking warfarin, 5 taking aspirin, and 2 taking clopidogrel. Among 46 patients with secondary ICH, 26 were classified into group 3 with vascular malformation (AVM, $n=21$; cavernous angioma, $n=2$; and dural AVF, $n=3$ ), 12 were classified into group 4 with brain metastasis (hepatocellular carcinoma, $n=7$; lung cancer, $n=3$; breast cancer, $n=1$; thyroid cancer, $n=1$ ), and 8 were classified into group 5 with primary brain tumors (glioblastoma, $n=6$; anaplastic astrocytoma, $n=1$; and ependymoma, $n=1$ ). Eighty-two of 110 cases were nonpathologically confirmed by complete disappearance of the hematoma in cases of primary $\mathrm{ICH}$ ( $n=57$ ); follow-up by CTA, MRA, or conventional angiography in cases of secondary ICH with vascular malformation $(n=18)$; and serial growing of the mass or enhancing tumor on follow-up imaging in cases of secondary ICH with tumor $(n=7)$.

Ages were significantly different among the 5 groups $(P<.01)$, and patients in group 3 were significantly younger than those in the other 4 groups in post hoc pair-wise comparison. Sex and time interval from symptom onset to CT were not significantly different.

\section{Histogram Analysis of ICH Attenuation}

Comparisons of histogram parameters among the 5 groups are listed in Table 2 and Fig 3, and representative cases of histogram analysis in tumorous and nontumorous ICH are shown in Fig 2. No significant correlation was observed between histogram parameters and symptom onset-CT time interval within 24 hours. In histogram analysis of

Table 1: Demographic data of study population $(n=110)$

\begin{tabular}{|c|c|c|c|c|c|c|}
\hline \multirow[b]{3}{*}{ Parameter } & \multicolumn{3}{|c|}{ Nontumorous ICH $(n=90)$} & \multicolumn{2}{|c|}{ Tumorous ICH $(n=20)$} & \multirow[b]{3}{*}{$P$ Value } \\
\hline & \multicolumn{2}{|c|}{ Primary ICH $(n=64)$} & \multicolumn{3}{|c|}{ Secondary ICH $(n=46)$} & \\
\hline & $\begin{array}{c}\text { Without } \\
\text { Antithrombotics } \\
(n=51)\end{array}$ & $\begin{array}{c}\text { With } \\
\text { Antithrombotics } \\
(n=13)\end{array}$ & $\begin{array}{c}\text { Vascular } \\
\text { Malformation } \\
(n=26)\end{array}$ & $\begin{array}{c}\text { Metastasis } \\
\quad(n=12)\end{array}$ & $\begin{array}{c}\text { Primary } \\
\text { Brain Tumor } \\
(n=8)\end{array}$ & \\
\hline Age (year) & $57.0 \pm 20.4$ & $66.8 \pm 11.7$ & $31.0 \pm 20.9$ & $56.8 \pm 14.9$ & $47.9 \pm 20.9$ & $<.01$ \\
\hline Gender (M:F) & $29: 22$ & $8: 5$ & 14:12 & $9: 3$ & $4: 4$ & .75 \\
\hline Onset-CT interval time (hours) & $10.0 \pm 8.2$ & $15.2 \pm 7.1$ & $8.5 \pm 7.8$ & $7.0 \pm 6.4$ & $9.5 \pm 7.1$ & .08 \\
\hline
\end{tabular}

${ }^{a}$ Difference among the 5 groups tested by 1-way analysis of variance or $\chi^{2}$ test. 
Table 2: Differences in histogram parameters for ICH attenuation among the 5 groups

\begin{tabular}{lcccccc}
\hline Parameter & $\begin{array}{c}\text { Primary ICH without } \\
\text { Antithrombotics }(\boldsymbol{n}=\mathbf{5 1})\end{array}$ & $\begin{array}{c}\text { Primary ICH with } \\
\text { Antithrombotics }(\boldsymbol{n}=\mathbf{1 3})\end{array}$ & $\begin{array}{c}\text { Vascular } \\
\text { Malformation }(\boldsymbol{n}=\mathbf{2 6})\end{array}$ & $\begin{array}{c}\text { Metastasis } \\
(\boldsymbol{n}=12)\end{array}$ & $\begin{array}{c}\text { Primary Brain } \\
\text { Tumor }(\boldsymbol{n}=\mathbf{8})\end{array}$ & $\begin{array}{c}\boldsymbol{P} \text { Value } \\
\mathbf{a}\end{array}$ \\
\hline Mean & $57.31 \pm 7.40$ & $52.64 \pm 7.35$ & $54.12 \pm 6.96$ & $46.94 \pm 9.07$ & $45.08 \pm 10.34$ & $<.01$ \\
Minimum & $21.65 \pm 9.65$ & $11.69 \pm 9.52$ & $18.62 \pm 7.05$ & $14.83 \pm 8.50$ & $9.00 \pm 5.29$ & $<.01$ \\
Percentiles & & & & & & \\
$\quad$ 5th & $37.73 \pm 6.70$ & $30.38 \pm 7.38$ & $35.56 \pm 5.53$ & $28.50 \pm 6.26$ & $26.38 \pm 6.50$ & $<.01$ \\
25th & $49.47 \pm 8.36$ & $42.77 \pm 9.14$ & $45.85 \pm 7.05$ & $38.58 \pm 8.50$ & $34.38 \pm 8.00$ & $<.01$ \\
50th & $58.78 \pm 8.46$ & $54.00 \pm 8.71$ & $55.35 \pm 8.31$ & $47.42 \pm 10.32$ & $44.38 \pm 11.65$ & $<.01$ \\
75th & $65.73 \pm 7.42$ & $62.92 \pm 6.71$ & $62.65 \pm 7.95$ & $55.00 \pm 10.57$ & $54.13 \pm 14.88$ & $<.01$ \\
$\quad$ 95th & $73.02 \pm 7.02$ & $71.15 \pm 5.51$ & $70.00 \pm 7.38$ & $64.58 \pm 9.02$ & $67.25 \pm 11.65$ & $<.01$ \\
Maximum & $99.33 \pm 16.46$ & $104.69 \pm 17.67$ & $94.31 \pm 18.47$ & $92.08 \pm 14.05$ & $122.00 \pm 65.95$ & .29 \\
Skewness & $-0.32 \pm 0.35$ & $-0.18 \pm 0.50$ & $-0.13 \pm 0.56$ & $0.05 \pm 0.47$ & $0.63 \pm 0.66$ & $<.01$ \\
Kurtosis & $2.64 \pm 0.52$ & $2.95 \pm 0.96$ & $3.00 \pm 2.11$ & $2.96 \pm 1.21$ & $4.11 \pm 2.47$ & $<.01$ \\
\hline
\end{tabular}

${ }^{a}$ Difference among the 5 groups tested using 1-way analysis of variance before pair-wise comparison.
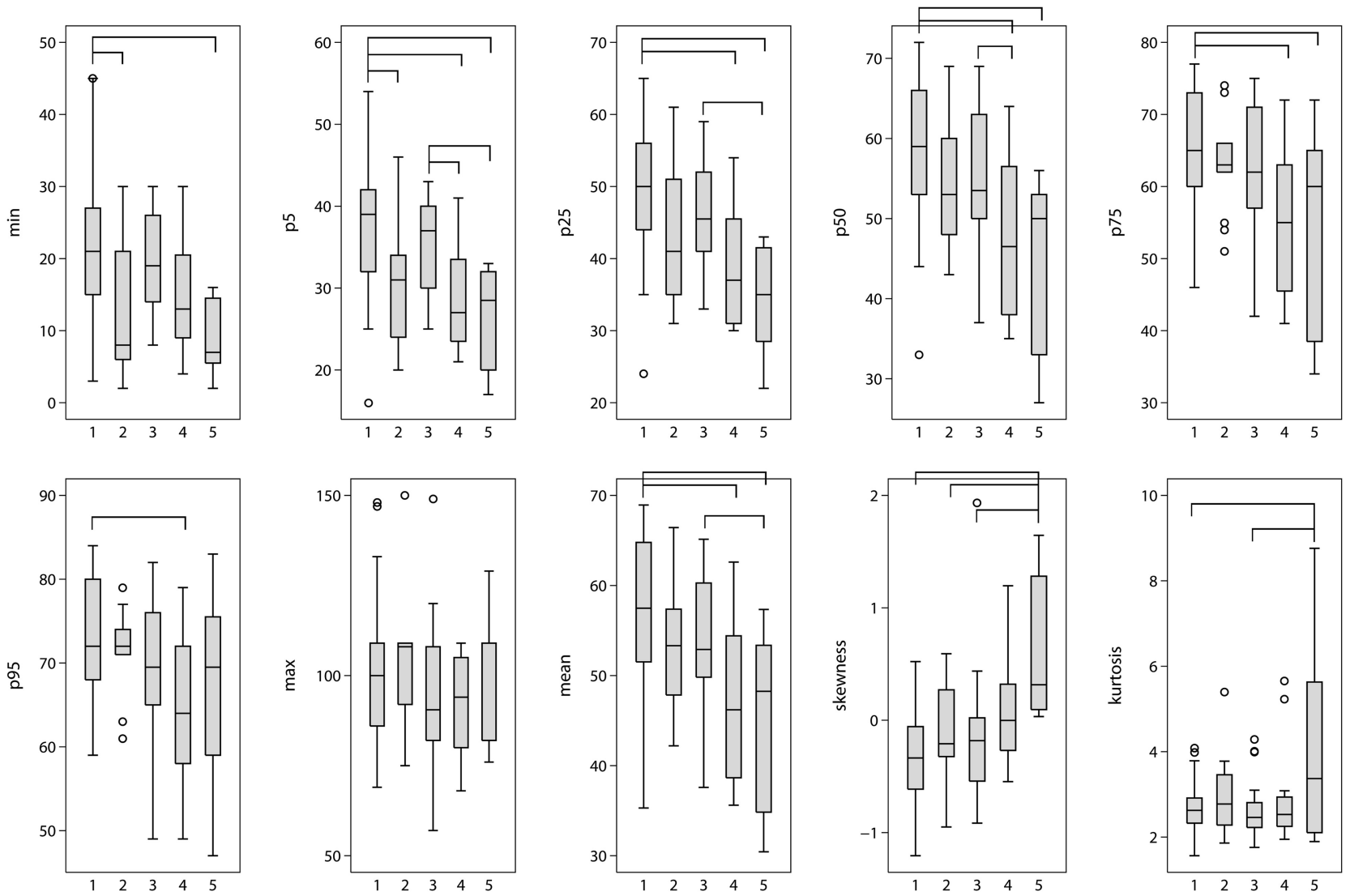

$\square=$ statistical significance

FIG 3. Differences among the 5 groups in histogram parameters of $\mathrm{ICH}$ attenuation are list on the $x$-axis of each boxplot: 1) primary ICH without antithrombotics, 2) primary ICH with antithrombotics, 3) secondary ICH due to vascular malformation, 4) secondary ICH due to brain metastasis, and 5) secondary ICH due to primary brain tumor.

ICH attenuation, group 1 was significantly higher than group 4 with brain metastasis and group 5 with primary brain tumor in the 5 th, 25th, 50th, and 75th percentile values. Group 1 was also significantly higher than group 4 in the 95th percentile value. For primary ICH, group 2, with patients taking antithrombotic medication, was significantly lower than group 1 for the minimum and 5th percentile values. Five of 13 patients of group 2 showed blood-fluid levels within the ICH, and all 5 of these patients were on warfarin. Group 3, with patients with vascular malformations, was significantly higher than groups 4 and 5 for 5 th percentile values and was higher than group 5 for 25 th and 50th percentile values. Maximum values were not significantly different among the 5 groups. Group 5 showed signifi- cantly higher kurtosis than groups 1 and 3. Groups 1,2, and 3 showed negative skewness, and groups 4 and 5 showed positive skewness with significant differences.

After dichotomization into tumorous and nontumorous ICHs, all histogram parameters except maximum value and kurtosis were significantly different between tumorous and nontumorous ICHs (Fig 4). Tumorous ICH had lower cumulative histogram parameters and was positively skewed.

\section{Receiver Operating Characteristic Analysis}

In receiver operating characteristic analysis (Table 3), all presumptive parameters distinguishing tumorous and nontumorous 

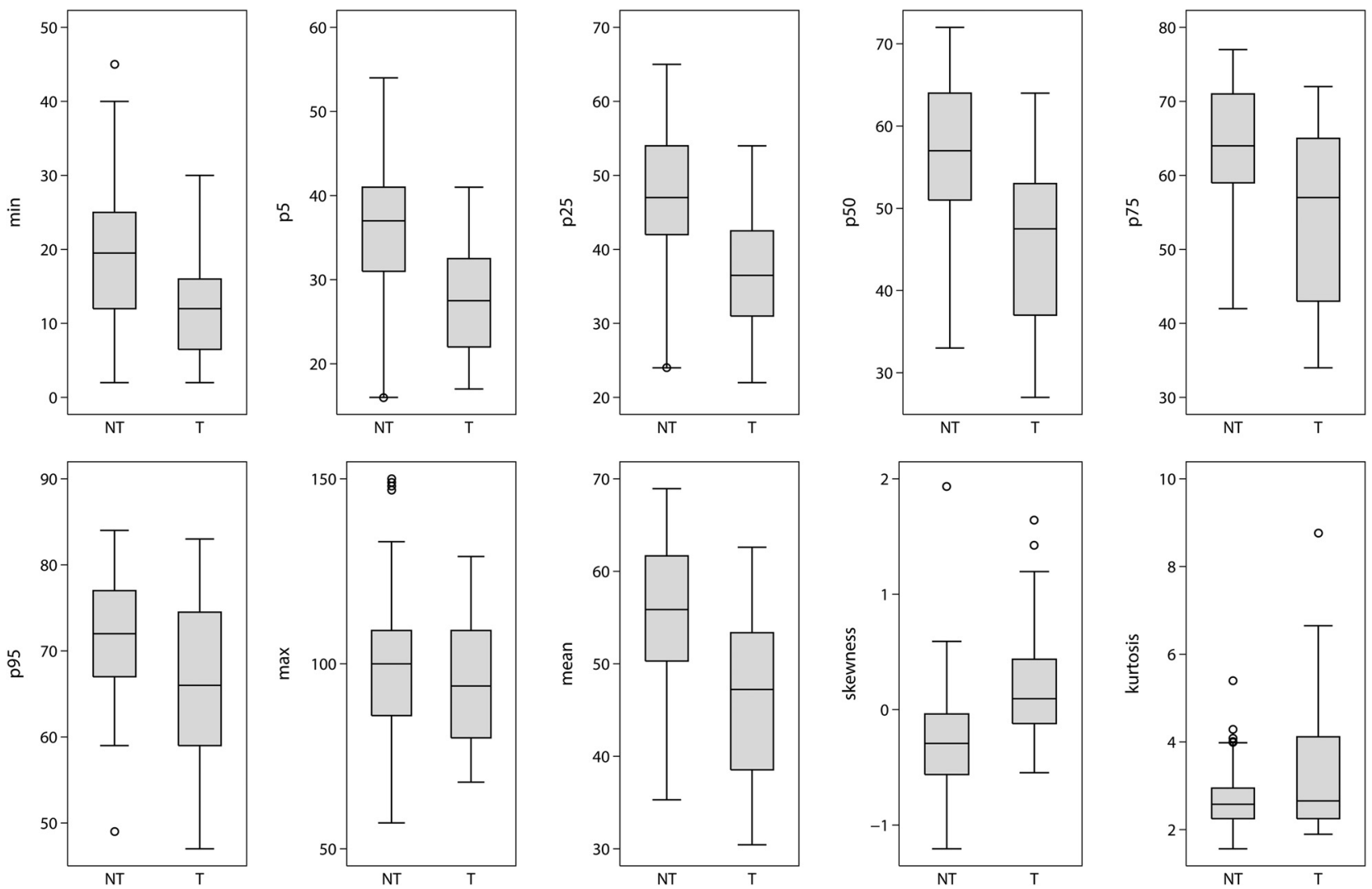

FIG 4. Differences in histogram parameters of ICH attenuation in tumor and nontumorous ICHs. All histogram parameters except maximum value and kurtosis were significantly different between tumorous and nontumorous ICHs. Tumors had lower cumulative histogram parameters and positive skewness. NT indicates nontumorous ICHs; T, tumorous ICHs.

Table 3: Diagnostic performance of ICH attenuation histogram parameters for discriminating tumor and nontumorous ICHs

\begin{tabular}{|c|c|c|c|c|c|}
\hline Parameter & $\begin{array}{c}\mathrm{Az} \\
\text { Value }\end{array}$ & $95 \% \mathrm{Cl}$ & $\begin{array}{l}\text { Cutoff } \\
\text { Value }^{\text {a }}\end{array}$ & $\begin{array}{c}\text { Sensitivity } \\
(\%)\end{array}$ & $\begin{array}{c}\text { Specificity } \\
(\%)\end{array}$ \\
\hline $\begin{array}{l}\text { Minimum } \\
\text { Percentiles }\end{array}$ & 0.71 & $0.59-0.84$ & 17 & 62.2 & 85.0 \\
\hline 5th & 0.81 & $0.72-0.91$ & 34 & 65.6 & 85.0 \\
\hline 25th & 0.81 & $0.71-0.92$ & 44 & 70.0 & 80.0 \\
\hline 50th & 0.78 & $0.66-0.89$ & 53 & 65.6 & 75.0 \\
\hline 75th & 0.74 & $0.60-0.87$ & 60 & 72.2 & 65.0 \\
\hline 95th & 0.69 & $0.54-0.84$ & 63 & 90.0 & 45.0 \\
\hline Mean & 0.78 & $0.66-0.90$ & 49.11 & 81.1 & 65.0 \\
\hline Skewness & 0.78 & $0.67-0.88$ & -0.02 & 75.0 & 25.0 \\
\hline
\end{tabular}

Note:-Az indicates area under the receiver operating characteristic curve. a Determined by maximizing the Youden index.

ICHs showed good diagnostic performance with area under the curve values above 0.7 (Fig 5). The 5 th and 25 th percentile values showed the highest diagnostic performance with area under the curve values of 0.81 and optimal cutoff values at $34 \mathrm{HU}$ and 44 HU, respectively, with $65.6 \%$ sensitivity and $85.0 \%$ specificity for the 5 th percentile value and $70.0 \%$ sensitivity and $80.0 \%$ specificity for the 25 th percentile value. The receiver operating characteristic curve of 5 th and 25 th percentile values showed no significant differences after adjusting for symptom onset to CT time interval, with age and sex as covariates.

\section{DISCUSSION}

For acute ICH, we investigated the ability of CT densitometry to discriminate between tumors and nontumorous causes of ICH. With
NECT only, tumorous and nontumorous ICHs were differentiated with good diagnostic performance by using histogram analysis of acute ICH attenuation. Among the examined histogram parameters, the 25th and 5 th percentile values had excellent diagnostic value with area under the curve values above 0.8 . The study population was confined to patients with lobar ICH, in whom secondary ICH is relatively frequent and primary lobar $\mathrm{ICH}$ is not negligible, accounting for $15 \%-25 \%$ of hypertensive spontaneous $\mathrm{ICH},{ }^{1,17}$ making determining a treatment strategy difficult.

Maximum value, unlike the other parameters, was not significantly different among the 5 groups and had poor diagnostic value. This result was because we set an upper limit of $130 \mathrm{HU}$ for thresholding to draw an ROI; maximum values were fixed at $130 \mathrm{HU}$ in case of hematomas adjacent to the skull causing partial volume artifacts. However, these pixels with partial volume artifacts account for a relatively small portion of the hematoma, so the other histogram parameters minimized this problem. Groups showing high attenuation by cumulative histogram parameters, such as groups 1 and 3 , showed relatively lower kurtosis. This result might be because highly attenuated acute hematoma had considerable interspersed lowattenuated voxels that were counted on voxelwise histogram analysis. In this context, the higher kurtosis with wider variance seen for group 5 might be attributable to a higher but variable proportion of tumor tissue with lower attenuation within the hematoma. After dichotomization, a higher trend for kurtosis was observed for tumorous than for nontumorous $\mathrm{ICH}$, though this result was not significant. 


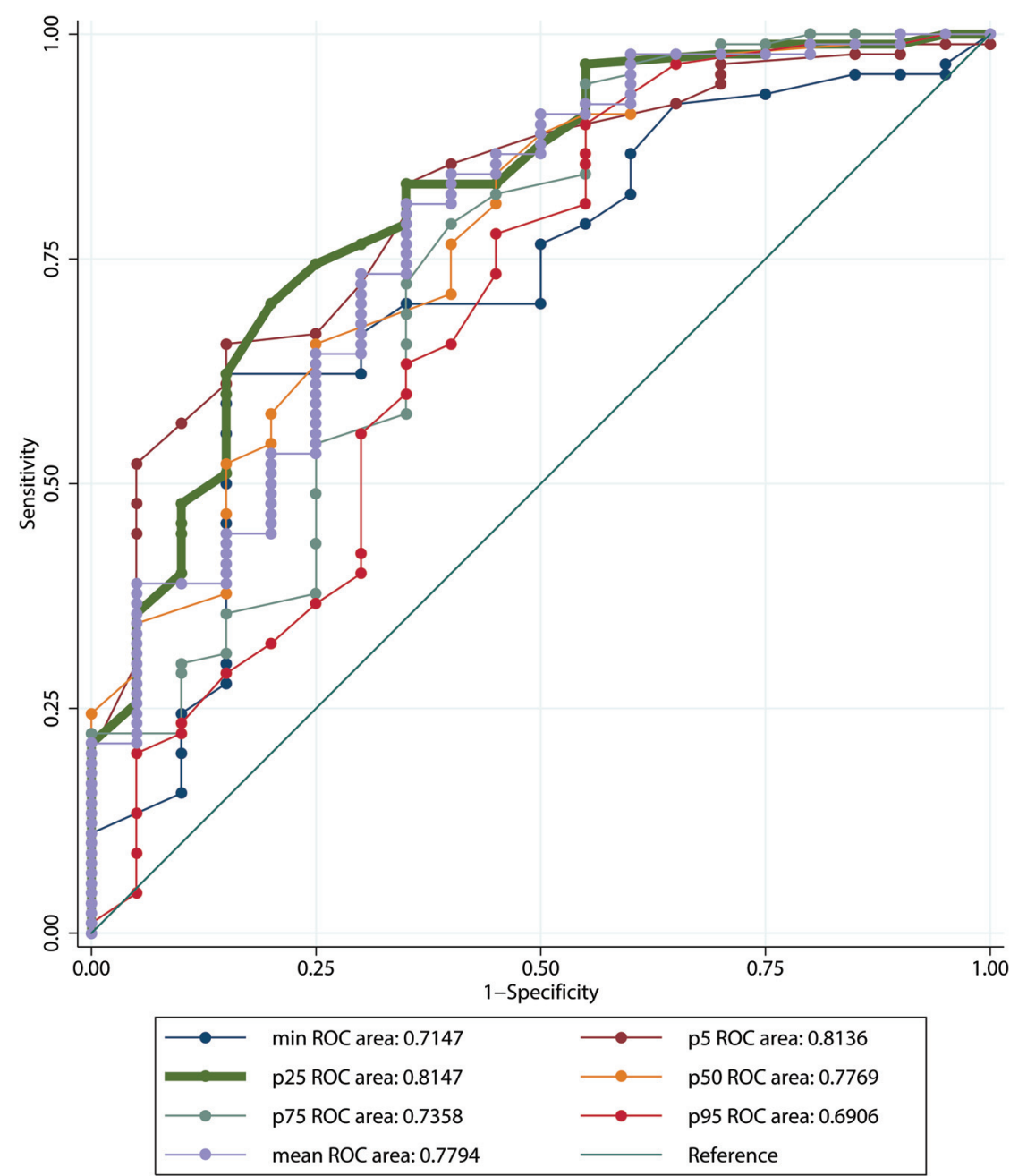

FIG 5. Receiver operating characteristic curves of histogram parameters for discrimination of tumor and nontumorous ICHs.

As shown in Fig 2, tumorous ICH showed a higher relative frequency of hematoma attenuation at lower Hounsfield unit values than nontumorous $\mathrm{ICH}$, leading to positive skewness and relatively lower Hounsfield unit values of tumorous ICHs for all cumulative histogram parameters except the maximum value. One possible explanation for this trend is that a solid tumor lesion within the hematoma might be clustered at lower Hounsfield unit values, though solid tumor lesions are difficult to detect by visual inspection after excluding patients with definitive tumorous $\mathrm{ICH}$ with gross cystic and solid lesions. Our receiver operating characteristic analysis showed the 5 th and 25 th percentile values to be the best parameters for differentiating the attenuation of hematomas between tumorous and nontumorous ICHs. We propose that histogram analysis is a useful tool for detecting hidden solid tumor lesions within acute $\mathrm{ICH}$, which might be helpful in establishing appropriate imaging follow-up and avoiding delays in long-term management of tumorous ICHs. Vascular malformation, another common cause of secondary $\mathrm{ICH},{ }^{6,7}$ showed relatively higher attenuation, similar to that of primary $\mathrm{ICH}$, and thus could be discriminated from tumorous ICH. One explanation for this result is that rupture of abnormal vessels is the main pathophysiology of acute bleeding. Therefore, as in primary $\mathrm{ICH}$, a solid lesion occupies a smaller proportion in vascular malformations than in tumors.

In the primary $\mathrm{ICH}$ group composed of groups 1 and 2, hematoma attenuation was lower in group 2 with antithrombotics, though this result was significant only for the minimum and 5 th percentile values. Five of 13 patients with antithrombotic medication showed blood-fluid levels in our study, and all 5 were on anticoagulants (warfarin). Lower attenuation at minimum and 5th percentile values might be because of this fluid-blood interface, which has been reported to result from uncondensed blood and can be seen in acute ICH of patients treated with anticoagulants within the first 12 hours. ${ }^{18,19}$ The lower and heterogeneous attenuation of primary ICH in patients treated with antithrombotics made differentiating primary ICH from tumorous ICH difficult. Therefore, clinicians should consider the possibility of misdiagnosis when examining acute ICH in patients with antithrombotic medication. In patients treated with anticoagulants, the fluid-blood interface uniquely seen in these patients might be helpful in diagnosis.

In routine clinical settings, prognosis and management of secondary ICH depend on the underlying etiology of $\mathrm{ICH}$, while those of primary ICH depend on hematoma growth, initial ICH volume, and expansion into the ventricles. ${ }^{20}$ Contrast-enhanced CT or MR imaging is performed when secondary $\mathrm{ICH}$, especially tumorous $\mathrm{ICH}$, is suspected. NECT is often undertaken serially to confirm resolution of primary ICH. However, high attenuation on CT and varying T1 intensity on MR imaging for acute hematoma can mask enhancing lesions of ICH on CT and MR imaging. ${ }^{11,12}$ Previous studies ${ }^{13,14}$ showed that dual-energy CT improves detection of enhancement within highly attenuated hemorrhage, but dual-energy CT or even single-energy contrast-enhanced CT and MR imaging are not always used as the initial imaging technique in patients with acute ICH. Serial follow-up with NECT takes time to confirm complete ICH resolution and might cause diagnostic delay. Being suspicious of tumor bleeding before complete ICH resolution might be helpful in determining appropriate imaging follow-up strategies and avoiding delays in long-term management of tumorous $\mathrm{ICH}$, though acute management would not differ. In this context, our study has value in that NECT is a standard technique initially performed on patients with acute ICH in routine clinical settings. To our knowledge, this is the first study investigating the use of NECT for discriminating tumorous and nontumorous ICHs by using CT densitometry. 
This study had several limitations. First, the CT protocols were heterogeneous because we included CT scans obtained at other hospitals in the study population. The voltage used in CT scans obtained at other hospitals was similar to that used for CT at our hospital, which was from 100 to $120 \mathrm{kV}$. The heterogeneous CT protocols probably did not affect our results because attenuation of a lesion changes depending on the voltage used in CT, and attenuation of the lesions would be similar if the voltages used in CT are similar. In addition, attenuation of solid lesions and hemorrhage does not change substantially as voltage increases, in contrast to the attenuation of iodine and calcification. ${ }^{13}$ A second limitation was that the ROIs in our study were subjectively drawn and could be regarded as cumbersome. However, semiautomatic methods of Hounsfield unit value thresholding has been used in previous studies ${ }^{16,21}$ and partially mitigated this limitation. In our experience, it took, on average, 8-10 minutes for an experienced neuroradiologist to segment and analyze a hematoma. Third, only a small number of patients with tumorous ICHs (groups 4 and 5) were included. In addition, the heterogeneity of primary malignancies in group 4 (secondary ICH with brain metastasis) is related to the heterogeneous cellularity of brain metastasis. Hence, it is likely for hypercellular brain metastasis to cause less low attenuation in hematomas and falsenegatives in the histogram analysis we proposed. This small number and heterogeneity of patients resulted from the strict exclusion criteria, including multiple hemorrhagic lesions, gross solid or cystic lesions, and the initial CT scan obtained $>24$ hours after symptom onset because we tried to include clinically confusing cases only. Nonetheless, the strict exclusion criteria can also be a limitation of our study because this study does not reflect the unselected patients who present with acute ICH in the emergency department. Fourth, the number of patients taking antithrombotic medications was relatively small, confined to the primary ICH group. Subsequently, the effect of antithrombotic medication was not fully stratified in all groups. This grouping was because all patients with secondary ICH taking antithrombotic medication were excluded due to other exclusion criteria. Nonetheless, patients taking antithrombotic medication tended to have lower attenuation in acute primary $\mathrm{ICH}$ in our study. If this is the case for tumorous $\mathrm{ICH}$, attenuation of tumor ICH with antithrombotic medication would be more likely opposite of expectations for primary $\mathrm{ICH}$; thus, antithrombotic medication would not affect the diagnostic performance of CT densitometry. However, the subgroup of patients taking antithrombotic medications included patients with both antiplatelets and anticoagulants, which also can be a limitation of our study. Patients treated with anticoagulants are more prone to have unclotted components in their hematomas, which might have contributed more to the lower hematoma attenuation, than patients taking antiplatelets. ${ }^{22,23}$ Fifth, no validation has been performed in this study, which needs further investigation.

\section{CONCLUSIONS}

We investigated the diagnostic value of CT densitometry for discriminating tumors and nontumorous causes of acute ICH. Histogram parameters for acute $\mathrm{ICH}$ attenuation were significantly different for tumorous and nontumorous ICHs. The 5th and 25th percentile values showed the highest diagnostic performance, with area under the curve values above 0.8 , sensitivities of $65.6 \%$ and $70.0 \%$, and specificities of $85.0 \%$ and $80.0 \%$, respectively. CT densitometry of acute ICH on NECT was a useful tool for discriminating tumorous and nontumorous ICH in patients with acute ICHs of unclear origin.

\section{REFERENCES}

1. Qureshi AI, Tuhrim S, Broderick JP, et al. Spontaneous intracerebral hemorrhage. N Engl J Med 2001;344:1450-60

2. Broderick JP, Brott T, Tomsick T, et al. The risk of subarachnoid and intracerebral hemorrhages in blacks as compared with whites. N Engl J Med 1992;326:733-36

3. Furlan AJ, Whisnant JP, Elveback LR. The decreasing incidence of primary intracerebral hemorrhage: a population study. Ann Neurol 1979;5:367-73

4. Sacco S, Marini C, Toni D, et al. Incidence and 10-year survival of intracerebral hemorrhage in a population-based registry. Stroke 2009;40:394-99

5. Foulkes MA, Wolf PA, Price TR, et al. The Stroke Data Bank: design, methods, and baseline characteristics. Stroke 1988;19:547-54

6. Scott M. Spontaneous intracerebral hematoma caused by cerebral neoplasms: report of eight verified cases. J Neurosurg 1975;42:338-42

7. Fewel ME, Thompson BG Jr, Hoff JT. Spontaneous intracerebral hemorrhage: a review. Neurosurg Focus 2003;15:E1

8. Destian S, Sze G, Krol G, et al. MR imaging of hemorrhagic intracranial neoplasms. AJR Am J Roentgenol 1989;152:137-44

9. Atlas SW, Grossman RI, Gomori JM, et al. Hemorrhagic intracranial malignant neoplasms: spin-echo MR imaging. Radiology 1987;164:71-77

10. Licata B, Turazzi S. Bleeding cerebral neoplasms with symptomatic hematoma. J Neurosurg Sci 2003;47:201-10; discussion 210

11. Inamasu J, Kuramae T, Nakatsukasa M. Glioblastoma masquerading as a hypertensive putaminal hemorrhage: a diagnostic pitfall. Neurol Med Chir (Tokyo) 2009;49:427-29

12. Meyer JR, Gorey MT. Differential diagnosis of nontraumatic intracranial hemorrhage. Neuroimaging Clin North Am 1998;8:263-93

13. Kim SJ, Lim HK, Lee HY, et al. Dual-energy CT in the evaluation of intracerebral hemorrhage of unknown origin: differentiation between tumor bleeding and pure hemorrhage. AJNR Am J Neuroradiol 2012;33:865-72

14. Phan CM, Yoo AJ, Hirsch JA, et al. Differentiation of hemorrhage from iodinated contrast in different intracranial compartments using dualenergy head CT. AJNR Am J Neuroradiol 2012;33:1088-94

15. Gildersleeve N Jr, Koo AH, McDonald CJ. Metastatic tumor presenting as intracerebral hemorrhage: report of 6 cases examined by computed tomography. Radiology 1977;124:109-12

16. Ko SB, Choi HA, Carpenter AM, et al. Quantitative analysis of hemorrhage volume for predicting delayed cerebral ischemia after subarachnoid hemorrhage. Stroke 2011;42:669-74

17. Caplan LR. Intracerebral haemorrhage. Lancet 1992;339:656-58

18. Hart RG, Boop BS, Anderson DC. Oral anticoagulants and intracranial hemorrhage facts and hypotheses. Stroke 1995;26:1471-77

19. Cervera A, Amaro S, Chamorro A. Oral anticoagulant-associated intracerebral hemorrhage. J Neurol 2012;259:212-24

20. Davis S, Broderick J, Hennerici M, et al. Hematoma growth is a determinant of mortality and poor outcome after intracerebral hemorrhage. Neurology 2006;66:1175-81

21. Ko SB, Choi HA, Parikh G, et al. Multimodality monitoring for cerebral perfusion pressure optimization in comatose patients with intracerebral hemorrhage. Stroke 2011;42:3087-92

22. Gökçe E, Beyhan M, Acu B. Evaluation of oral anticoagulant-associated intracranial parenchymal hematomas using CT findings. Clin Neuroradiol 2014 Jan 29. [Epub ahead of print]

23. Pfleger MJ, Hardee EP, Contant C, et al. Sensitivity and specificity of fluid-blood levels for coagulopathy in acute intracerebral hematomas. AJNR Am J Neuroradiol 1994;15:217-23 